\begin{tabular}{|c|c|c|c|}
\hline$\Omega$ & 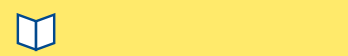 & $\equiv$ & (2) \\
\hline $\begin{array}{l}\text { Avinash Aujayeb, } \\
\text { Jonathan Miller, } \\
\text { lain Matthews }\end{array}$ & $\begin{array}{l}\text { Respiratory Department, } \\
\text { Wansbeck General } \\
\text { Hospital, Newcastle upon } \\
\text { Tyne, UK }\end{array}$ & $\begin{array}{l}\text { A. Aujayeb, Respiratory } \\
\text { Department, Wansbeck } \\
\text { General Hospital, } \\
\text { Woodhorn Ln, Ashington, } \\
\text { Northumberland NE63 9lJ, } \\
\text { UK }\end{array}$ & aujayeb@doctors.net.uk \\
\hline
\end{tabular}

\title{
Acute breathlessness in lung cancer
}

\section{Case Report}

\section{Case history}

A 72-year-old woman with a World Health Organization (WHO) performance status of 1 presented in October 2013 with a left upperlobe mass (fig. 1). She was an ex-smoker and a computed tomography (CT) scan showed a large mass at the left hilum (fig. 2) as well as a pulmonary embolus and a positron emission tomography (PET) scan staged the disease at $\mathrm{T}_{4} \mathrm{~N}_{3} \mathrm{M}_{1} \mathrm{~b}$, with contralateral mediastinal lymphadenopathy and pelvic bone metastases. Biopsies from bronchcoscopy showed an adenocarcinoma and epidermal growth factor receptor (EGFR) mutation was negative. She underwent four cycles of palliative chemotherapy with good radiological and clinical response (fig.3) and was started on warfarin.

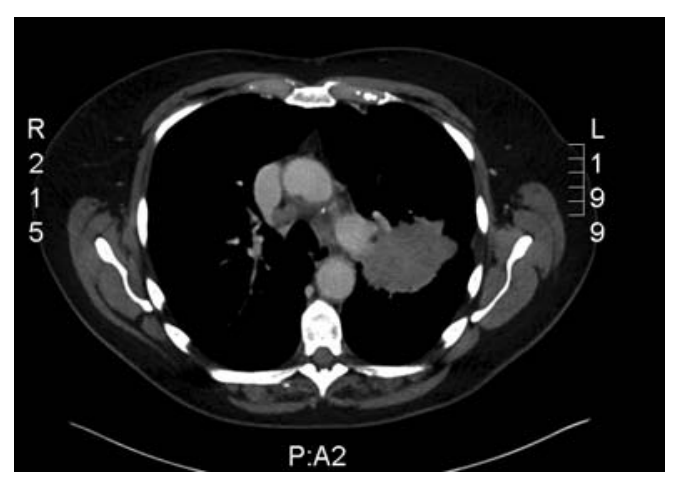

Figure 2

CT showing large mass at the left hilum

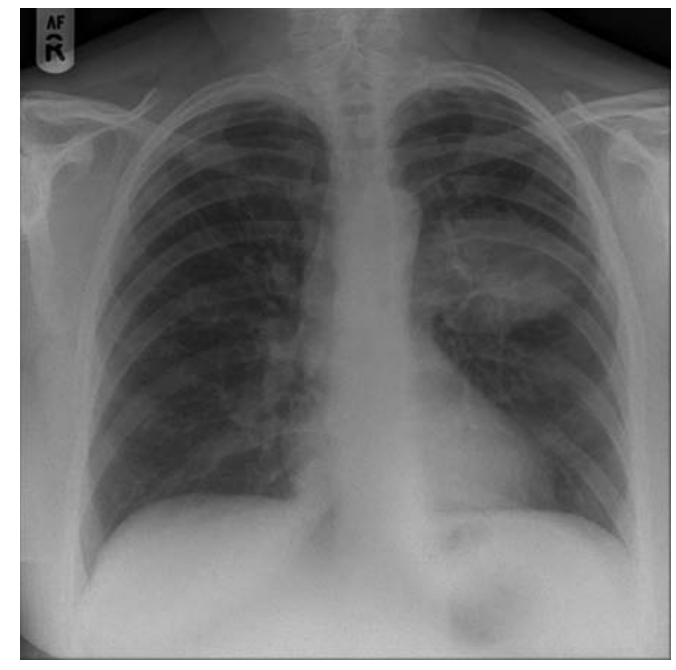

Figure 1

Initial presentation showed a left upper-lobe mass

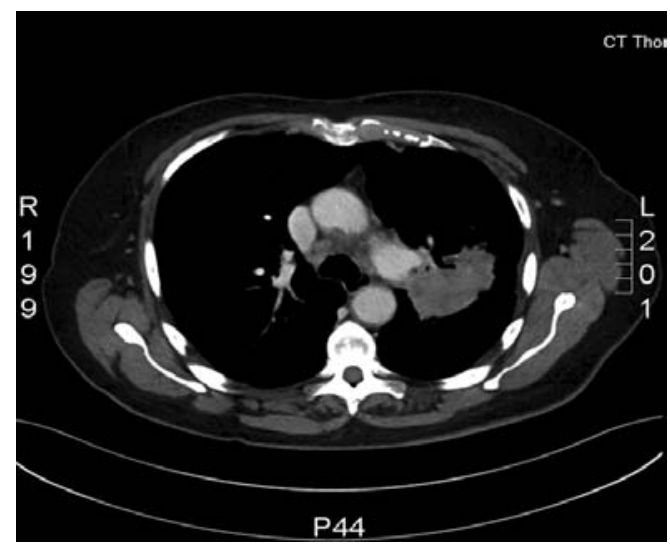

Figure 3

After four cycles of chemotherapy, there was good response
Statement of Interest None declared.
HERMES syllabus link: module D.3.1. 
At follow-up in August 2014, progressive disease was noted radiologically (figs 4,5 and 6) and palliative radiotherapy was thus considered for vertebral pain and then second-line chemotherapy.

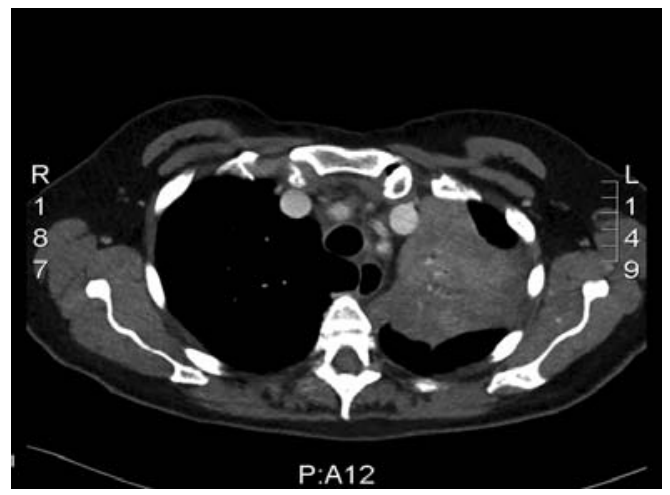

Figure 4

CT at follow-up

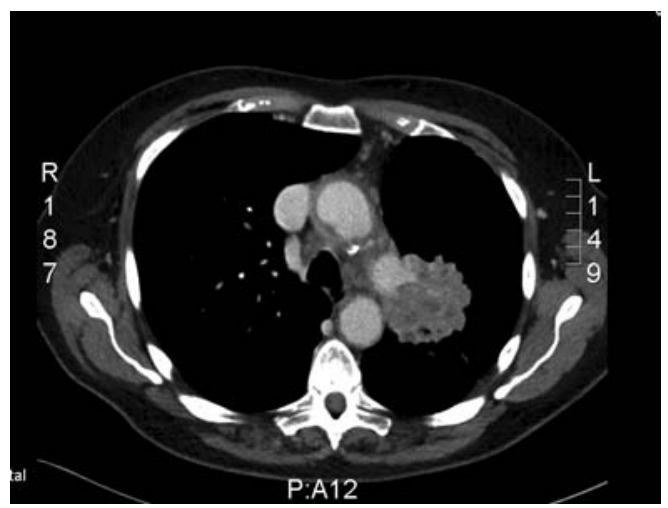

Figure 5

CT at follow-up

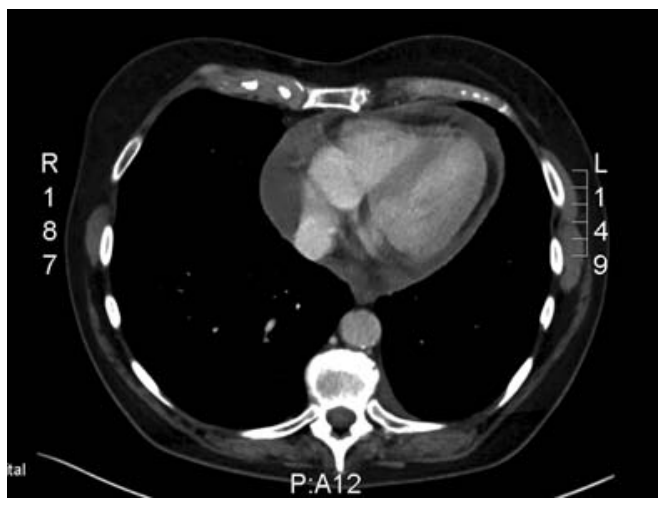

Figure 6

CT at follow-up

\section{Task 1}

What do the computed tomography images show (figs 4,5 and 6) ?

1. A small left effusion with an enlarging mass at the left hilum

2. A small pericardial effusion

3. Some degree of left upperlobe collapse

4. All of the above 


\section{Answer 1}

4. All of the above

She presented to the ambulatory care department via primary care with a high international normalisation ratio, probably due to the recent prescription of antibiotics for a presumed lower respiratory tract infection. At that point, she complained of increasing breathlessness and a chest radiograph was performed. Her oxygen saturation was $89 \%$ on $60 \%$ oxygen via a Venturi mask, she was normotensive but tachycardic at 130 beats $\cdot \mathrm{min}^{-1}$. Electrocardiography showed a sinus tachycardia. Her white cell count was normal and inflammatory markers such as C-reactive protein only mildly raised at $31 \mathrm{mg} \cdot \mathrm{L}^{-1}$. Her international normalised ratio (INR) was 2.3. Her chest was clear to auscultation and her heart sounds were normal. She was treated empirically for a presumed pneumonia with intravenous fluids and antibiotics.

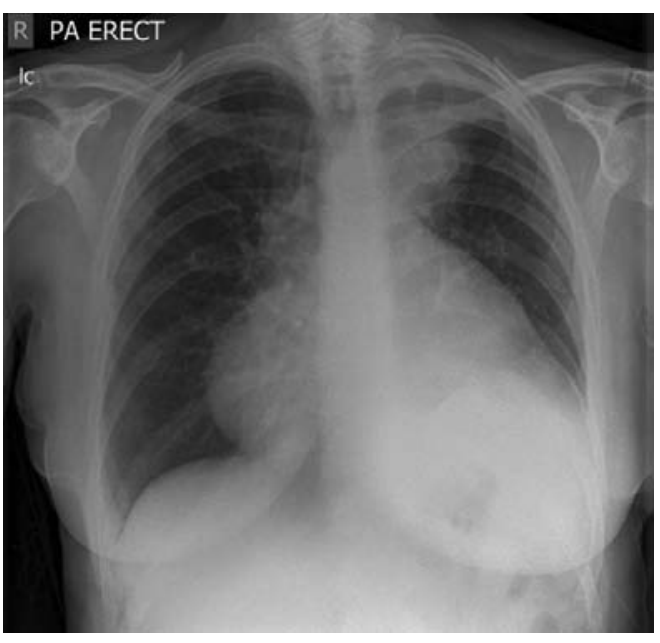

Figure 7

Chest radiograph at admission to the ambulatory care department

\section{Task 2}

What new finding does the admission chest radiograph in figure 7 show?

1. Significant right middle lobe consolidation

2. A left-sided pneumothorax

3. A large pericardial effusion

4. All of the above 


\section{Answer 2}

3. A large pericardial effusion

\section{Task 3}

What is the likely cause of her pericardial effusion?

1. Pericardial bleeding due to high INR

2. Atypical infection due to immunosuppression

3. Malignant disease

4. Autoimmune disease

\section{Answer 3}

3. Malignant disease

In areas of low tuberculosis prevalence, such as the North East of England, where our patient lived, large pericardial effusions are commonly due to malignancy. In various studies, the prevalence of malignant disease in pericardial disease was up to $60 \%$ [1-4]. Lung cancer is the most common disease causing malignant pericardial effusion (MPE) although case reports of breast and oesophageal cancers, leukaemia, lymphomas and melanomas exist [2]. Large autopsy studies of patients dying of metastatic cancer showed that the prevalence of any cardiac involvement is $11-20 \%$ and, of those, about $30 \%$ had a pericardial effusion [5]. Development of an MPE suggests metastatic disease via direct or indirect spread. However, other causes need to be kept in mind in a patient with cancer developing a pericardial effusion such as thoracic irradiation causing a radiationinduced pericarditis and subsequent fluid build-up or immunocompromise due to chemotherapy predisposing to infective aetiologies.

A diagnosis of cardiac tamponade was thus made.

\section{Task 4}

What signs cannot be expected to be seen in a patient with cardiac tamponade?

1. Collapsing pulse

2. Pulsus paradoxus

3. Pulsus alternans

4. Quiet heart sounds 


\section{Answer 4}

1. Collapsing pulse

Cardiac tamponade arises due to equalisation of cardiac pressures leading to impaired biventricular filling. The pericardial sack has some elasticity which is quickly reached with fluid accumulation. Cardiac volume becomes smaller during systole and venous return shifts to cardiac systole to reduce cardiac output. Normally, systemic venous return to the right side of the heart increases with inspiration and pulmonary venous return to the left heart decreases with inspiration so that systemic arterial pressure normally falls by $<10 \mathrm{mmHg}$ during inspiration.

In cardiac tamponade, the rigid pericardium prevents the free wall from expanding. Thus, the distension of the right ventricle is limited to the interventricular septum and this causes the septum to bulge to the left, reducing left ventricular compliance and contributing to further decreased filling of the left ventricle during inspiration. This leads to an exaggerated drop in systolic blood pressure in inspiration called pulsus paradoxus. Our patient was too sick to allow the proper measurement of this sign.

Thus, most of her symptoms and signs (breathlessness, tachycardia, hypotension and decreased capillary refill time peripherally) could be directly attributable to pericardial tamponade. The electrocardiogram showed small complexes but no pulsus alternans, which is where the QRS complexes alternate between large and small with each beat.

The patient was admitted to the local hospital, where there was no cardiology cover out of hours. Her high INR also precluded her having an immediate intervention. She was given Vitamin K, an antagonist of warfarin, oxygen and fluids and was thus optimised for a drain the next day.

\section{Task 5}

What is not the next appropriate step?

1. Urgent referral to cardiology for a pericardial drain

2. Oxygen to keep saturations between 88-92\%

3. Discussion with family and patient re escalation of care and do not resuscitate order

4. Reversal of high INR with vitamin $\mathrm{K}$ 


\section{Answer 5}

1. Urgent referral to cardiology for a pericardial drain

MPEs carry a very poor prognosis. When due to lung cancer, some series have put the life expectancy at 3 months [6] Our patient's WHO performance status had deteriorated significantly to 4 (WHO performance status of 4 means that the patient is bed-bound and cannot carry out self care) and, as such, at that moment in time, her risk of mortality was very high. As such, a treatment escalation plan was put in place and a do not resuscitate order was instituted. The patient and her family were in full agreement. Her INR was too high for any procedure to be performed and so $2 \mathrm{mg}$ of vitamin $\mathrm{K}$ was given intravenously. Our hospital is a district general hospital with no cardiology cover out of hours, and she had presented on a Sunday, but we did not think that an urgent cardiology opinion should be sought from the tertiary centre.

The patient was thus admitted locally. Her high INR also precluded her having an immediate intervention. She was given vitamin $\mathrm{K}$, an antagonist of warfarin, oxygen and fluids and was thus optimised for a drain the next day.

\section{Conclusion}

The next day, an echocardiogram showed a large pericardial effusion and with an INR of 1.8, a pericardial drain was inserted. $2 \mathrm{~L}$ of heavily blood-stained fluid was drained, with significant improvement in the patient's haemodynamic and respiratory status over the next $24 \mathrm{~h}$. She was eventually discharged

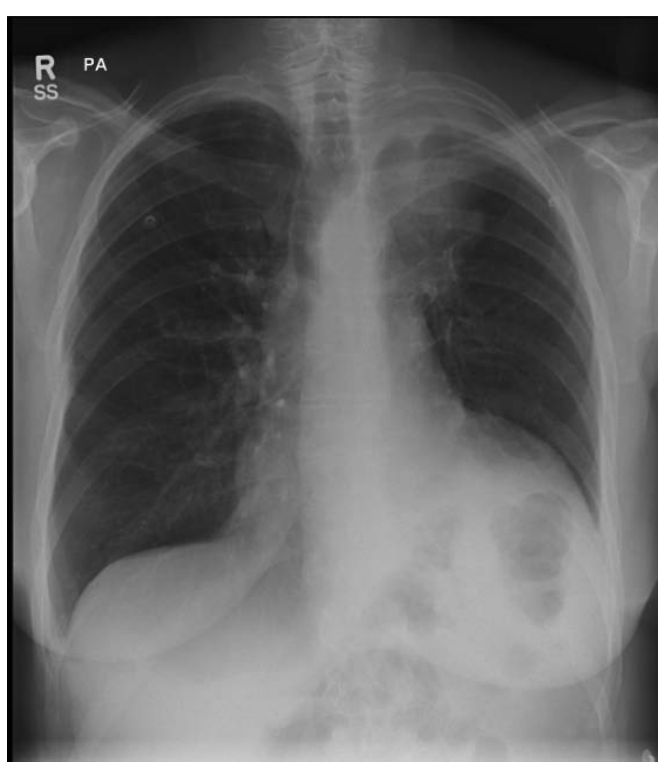

Figure 8

Radiograph just prior to discharge

home $48 \mathrm{~h}$ later, not requiring any oxygen and with a WHO performance status of 2 (which means that a patient is symptomatic but is up and about more than $50 \%$ of waking hours). Figure 8 shows her chest radiograph just prior to discharge.

Recurrence of her MPE was briefly discussed and it was felt that she was not suitable for referral for a pericardial window at this moment in time. We felt the effusion was malignant as its development was noted in hindsight on the previous CT scans rather than a reactive one due to her over anticoagulation. Unfortunately, no fluid was sent for cytology testing. 4 weeks after her discharge, a marked deterioration was noted by the patient's general practitioner and full palliative measures were instituted. The patient passed away peacefully in her own home.
4. Porte HL, Janecki-Delebecq TJ, Finzi L, et al. Pericardoscopy for primary management of pericardia effusion in cancer patients. Eur / Cardiothorac Surg 1999; 16: 287-291.

5. Abraham KP, Reddy V, Gattuso P. Neoplasms metastatic to the heart: review of 3314 consecutive autopsies. Am J Cardiovasc Pathol 1990; 3: 195-198.

6. Cullinane CA, Paz IB, Smith D, et al. Prognostic factors in the surgical management of pericardial effusion in the patient with concurrent malignancy. Chest 2004 125: $1328-1329$. patients with pericardial effusion. J Clin Oncol 2005: 23: $5211-5216$ 